\title{
Modeling and Stability Analysis of a Three Species Ecosystem with the Third Species Response to the First Species in Sigmoid Functional Response Form
}

\author{
Geremew Kenassa Edessa, Purnachandra Rao Koya \\ Department of Mathematics, Wollega University, Nekemte, Ethiopia \\ Email address: \\ gbonsa.kena@gmail.com (G. K. Edessa),drkpraocecc@yahoo.co.in (P. R. Koya)
}

\section{To cite this article:}

Geremew Kenassa Edessa, Purnachandra Rao Koya. Modeling and Stability Analysis of a Three Species Ecosystem with the Third Species Response to the First Species in Sigmoid Functional Response Form. Mathematical Modelling and Applications.

Vol. 5, No. 3, 2020, pp. 156-166. doi: 10.11648/j.mma.20200503.14

Received: May 1, 2020; Accepted: June 18, 2020; Published: August 4, 2020

\begin{abstract}
In this paper, a three species eco system, involving three pairs is considered modeled to examine the stability. Among the three species, one plays dual roles which are a host and an enemy with Monod response. In the first place model assumptions and formulation was carried out for investigations. The biological feasibility of the system is checked. That is positivity and boundedness of the model is verified. It is shown that biologically valid. The dynamical behavior of the proposed model system was analyzed qualitatively. The dynamical analysis includes the determination of all possible equilibrium points and their stability properties. All the equilibrium states are identified and the local asymptotic stability of some of the equilibrium states is examined by considering the set criteria. It is observed that among the states, the state in which the Prey and its Host species are exist is stable and the state where the Predator/Ammensal species is washed out is asymptotically stable. The global stability of the co-existence of the species was investigated by constructing a suitable Lyapunov function. To support our analytical studies, some numerical simulations was performed susing some mathematical software and the results were forwarded in the last section.
\end{abstract}

Keywords: Prey, Predator, Ammensal, Commensal, Host, Continuous Time, Stability, Numerical Simulation

\section{Introduction}

The development of the qualitative analysis of ordinary differential equations is deriving to study many problems in mathematical biology.

Ecosystems are the ones in which their living and nonliving components interact with and depend on each other linking together the exchange of energy, material, information. The structure and the function of the ecosystems are determined by the interplay of both cooperation and competition [21, 22]. Ecosystems are able to regulate themselves to maintain certain stability. Therefore, the stability is one of the most fundamental and essential features of the ecological systems. The study of stability is directed relevant to the existence of every species. The stability is influenced by many factors, such as the structure within the components and the features of the environment. The ecosystems are complex and involve many kinds of interactions among the elements. The inherent interactions are often non-linear and intricate. These systems can be described by a set of nonlinear differential equations. These nonlinear interactions lead to complex dynamics. There have been many investigations on the stability of ecosystems. Most of the works have been focused on the local linear stability analysis. The studies of the stability of ecosystems are significant for uncovering the underlies ecological law of species and populations [34].

The global stability of the ecological systems is still challenging in general. Furthermore, the link between the global characterization of the ecological systems and the dynamics of the elements is still not clear. The past researchers explored the dynamical system with the approach of Lyapunov function which was developed to investigate the global stability. Here, in recent work we would like to suggest a universal and straightforward approach to explore the Lyapunov function and therefore the global stability of the general ecological systems. 
In nature, all living species like a suitable environment where it can live freely and reproduce. Ecological species take various techniques for searching foods and for defensive purposes.

Ecology, basically the study of the inter relationship between species and their environment, in such areas as predator-prey and competition interactions, renewable resources management, evolution of pesticide resistant strains ecological and genetically engineered control of pests, multi-species societies, plant-herbivore systems and so on is now an enormous field. It is the scientific study of the interactions between organisms and their environment [2325].

In the ecosystems, the relationship between species can be grouped into two categories: the negative antagonism interaction (-) and the positive mutualism interaction. Predation shows the relationship $(+/-)$ which one species is disfavored, while the other species benefits. Examples in the natural world include sharks and fish, lynx and snowshoe hares, and ladybirds and aphids. Mutualism shows the relationship $(+/+)$ which both species benefit from interactions of the other. Stability and dynamics are crucial for understanding the structure and the function of ecosystems [23, 29-34].

In this paper we are interested in ecological systems in which the interactions were both positive and negative to show the stability of the ecosystem.

A brief description of a commensalism interaction was given by different scholars [26-29, 31-32, 34].

Ammensalism is the ecological interaction in which an individual species harms another without obtaining benefit. This type of symbiotic relationship is common, but not considered an important process structuring communities because they are "accidental" and do not benefit the species doing the harm. It is a 0- relationship. For instance, algal blooms can lead to the death of many species of fish and other animals, however the algae do not benefit from the deaths of these individuals.

These different theoretical studies was manifested or visualized by applying mathematical language and defined by varies researcher [4-5, 8-9, 11-17].

Dynamics of non-linear systems that occur in ecological systems has attracted the attention of mathematicians since the days of Lotka [1] and Volterra [2]. Over the years, this model has attracted attentions for exploring the dynamical process of the ecology. Non-linear dynamic models exhibit a wide range of behaviors. The Lotka-Volterra Prey-Predator model involves two equations, one which describes how the prey population changes and the second which describes how the predator population changes would be defined by the differential equations as follow;

$$
\begin{gathered}
\frac{d H}{d t}=a H(t)-b H(t) P(t \\
\frac{d P}{d t}=e b H(t) P(t)-c P(t)
\end{gathered}
$$

Where a, b, c and e are all positive constants, with $H(t)$ and $P(t)$ representing the scaled population of prey and predator, respectively, and $t$ is measured in years.
Moreover; the other models involving Commensal-host and ammensalism model were described as shown in equation (2) and (3) respectively.

Commensal-host model:

$$
\begin{aligned}
& \frac{d H}{d t}=r H(t)\left[1-\frac{H(t)}{k}\right] \\
& \frac{d C}{d t}=a C(t)\left[1-\frac{C(t)}{k^{\prime}}\right]+e H(t) C(t)
\end{aligned}
$$

Where $r, k, k^{\prime}, a$ and $e$ positive constants with are $H(t)$ and $C(t)$ denotes the population of host and Commensal respectively.

Ammensalism model:

$$
\begin{gathered}
\frac{d N}{d t}=r N(t)\left[1-\frac{N(t)}{k}\right] \\
\frac{d A}{d t}=a A(t)\left[1-\frac{A(t)}{k^{\prime}}\right]-g N(t) A(t)
\end{gathered}
$$

Where $r, k, k$, $a$ and $g$ are positive constants with $N(t)$ and $A(t)$ are density of populations where $A(t)$ is Ammensal.

Inspired by these model, several researchers made significant contributions in this area by considering various special types of interactions between the species. This has been the motivation for others in bringing a third species into the system thus forming a three species ecological system.

In the present paper, the three species Ecosystem with time as continuous unit is considered. The equilibrium states are identified and the asymptotic stability of the equilibrium states is examined. A few of them are presented here.

Now, the present investigation is a study of a continuous model of "a symbiotic interaction and predation" between three species.

\section{Assumptions and Models Equations}

Mathematical modeling and computer simulation provide an effective tool in the study of contemporary population ecology [18, 19]. In population dynamics, the functional response of predator to prey density refers to the change in the density of prey attacked per unit time per predator as the prey density changes [20].

Recently, Koya P. Roa and Geremew K [34] worked on three species system by considering interactions like PreyPredator, Commensal - Host, and between the three species, which motivated the present authors to consider a three species Ecosystem with species S1, S2 and S3 simultaneously having the interactions of Prey-predation, commensalism and ammensalism, with continuous time. Here, S1 and S2 form a Prey-Predator pair. That is, S2 depends on S1 for its survival. S1 and S3 form a Commensal - Host pair. That is, S3 acts as host to S1 without itself being affected. Moreover, the response between S3 and S1 is sigmoid functional response not a linear. And S2 and S3 form an Ammensal - Enemy pair. That is, S3 inhibits S2 without itself being affected, as shown illustrated in figure 1 below.

In this paper, we describe the three species ecosystem 
model. To develop this model, the assumptions have been made as mentioned

The three model equations for $\mathrm{dN} 1 / \mathrm{dt}, \mathrm{dN} 2 / \mathrm{dt}$ and $\mathrm{dN} 3 / \mathrm{dt}$ are constructed using several components, both variables and parameters, and each of which represents specific biological assumptions.

The simple schematic interactions among the model variable is shown in Figure 1.

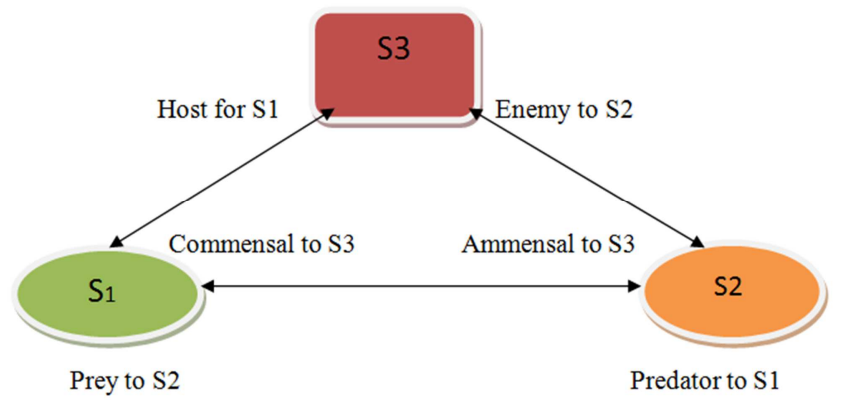

Figure 1. A three species ecosystems.

\subsection{The Model Equations}

Based on the above initial models given in system (1) - (3) and the assumptions, the interactions of the three species stated by the model equations as follows;

$$
\begin{gathered}
\frac{d N_{1}}{d t}=a_{1} N_{1}-a_{11} N^{2}{ }_{1}+\left[\frac{a}{1+a h N_{1}}\right] N_{1} N_{3}-a_{12} N_{1} N_{2} \\
\frac{d N_{2}}{d t}=a_{2} N_{2}-a_{22} N^{2}{ }_{2}+a_{21} N_{1} N_{2}-a_{23} N_{2} N_{3} \\
\frac{d N_{3}}{d t}=a_{3} N_{3}-a_{33} N^{2}{ }_{3}
\end{gathered}
$$

with initial conditions

$$
\begin{aligned}
& N_{1}(0)=N_{01}>0 \\
& N_{2}(0)=N_{02}>0 \\
& N_{3}(0)=N_{03}>0
\end{aligned}
$$

\begin{tabular}{|c|c|}
\hline Notation & Descriptions \\
\hline$a_{i}$ & $\begin{array}{l}\text { The Natural growth rate of } \mathrm{Si}, \mathrm{i}=1,2,3 \text {; the difference of } \\
\text { birth and death rate. }\end{array}$ \\
\hline$a_{i i}$ & $\begin{array}{l}\text { Self inhibition coefficient of } \mathrm{Si}, \mathrm{i}=1,2,3 \text {. (The rate of } \\
\text { decrease of } \mathrm{Ni} \text { due to insufficient natural resources of } \mathrm{Si} \text { ) }\end{array}$ \\
\hline$a_{12}$ & The rate of decrease of S1 due to inhibition by S2 \\
\hline$A$ & The functional response of the $\mathrm{S} 1$ to its host $\mathrm{S} 3 ; \mathrm{A}=$ \\
\hline$a_{21}$ & $\begin{array}{l}1+a h N_{1} \\
\text { The rate of increase of the } \mathrm{S} 2 \text { due to its attacks on } \mathrm{S} 1\end{array}$ \\
\hline$a_{23}$ & $\begin{array}{l}\text { The rate of decrease of the S2 due to the harm caused by } \\
\text { its enemy S3 }\end{array}$ \\
\hline $\begin{array}{l}a_{12} / a_{11} \\
A / a 11\end{array}$ & $\begin{array}{l}\text { Coefficient of prey/commensal inhibition of the predator } \\
\text { Coefficient of commensalism }\end{array}$ \\
\hline$a 21 / a 22$ & Coefficient of predator consumption of the prey \\
\hline$\frac{a 23}{a 22}$ & Coefficient of Ammensalism \\
\hline
\end{tabular}

Notations and descriptions of parameters

Notations and descriptions of the state variables and parameter are listed in the table 1 below.

Table 1. Notation and description of the model parameters.
The model variables $N i(t)$ the density of population $\mathrm{Si}$, $\mathrm{i}=1,2,3$ at any instant of time $\mathrm{t}$ subject to the non-negative initial conditions $N i(0)=N_{0}>0$.

Moreover, the notation $\mathrm{A}$ in the model indicates the Functional response with Holling type II response, i.e., $A=$ $\frac{a}{1+a h N_{1}}$, where a is helping rate, h-handling rate.

\section{Dynamical Properties of the Model System}

Since the state variables $N_{1}, N_{2}$ and $N_{3}$ represent population sizes, positivity implies that the population sizes never become negative. The boundedness of the system is interpreted as a natural restriction to the growth of populations as consequences of limited resources.

Positivity and boundedness of the solution of the system

In this section, some basic dynamical properties of the system are discussed subjected to positive initial conditions.

Positivity of the solution

Here the positivity of each population size such as $N_{1}(t)$, $N_{2}(t)$ and $N_{3}(t)$ is verified. These system variables must have the positive values in order to be biologically meaningful. The positivity of these biological or system variables is tested and the results are presented in the form of proposition as follows:

Proposition 1 Every solution of system (4-6) together with the positive initial conditions exists in the interval $[0, \infty)$ and they are non-negative. That is, $N_{1}(t), N_{2}(t)$ and $\left.N_{3}(t) \geq 0\right)$ for all $t \geq 0$.

\section{Proof}

For $t \in[0, T]$, as the system (4) is continuous, then the solution $N_{1}(t), N_{2}(t)$ and $N_{3}(t$ of the system with given initial conditions exists and unique on $[0, T]$ where $0<T<$ $+\infty$.

a) Positivity of $N_{1}(t), N_{2}(t)$ and $N_{3}(\mathrm{t})$

Verifying the positivity of $N_{1}(t)$ : The density of the first population of the system (2.1) is solved analytically and its solution is obtained as:

$$
N_{1}(t)=N_{01} \exp \int_{0}^{T}\left[a_{1}-a_{11} N_{1}(t)+\frac{a N_{3}(t)}{1+a h N_{1}}-a_{12} N_{2}\right] d t
$$

The exponential function is always non-negative and the initial population $N_{1}(t)$ is assumed to be positive. Therefore, $N_{1}(t)>0$ for all $\mathrm{t} \geq 0$.

Verifying the positivity of $N_{2}(t)$ : The density of the second population of the system (5) is solved analytically and its solution is obtained as follow:

$$
\begin{aligned}
N_{2}(t)=N_{02} & {\left[\operatorname { e x p } \int _ { 0 } ^ { T } \left(a_{2}-a_{22} N_{2}(u)+a_{21} N_{1}(u)\right.\right.} \\
& \left.\left.-a_{23} N_{3}(u)\right)\right] d u
\end{aligned}
$$

The exponential function is always non-negative and the 
initial population $N_{02}$ is assumed to be positive. Therefore, $N_{2}(t)>0$ for all $\mathrm{t} \geq 0$.

Verifying the positivity of $N_{3}(\mathrm{t})$ : The density of the third population of the system (6) is solved analytically and its solution is obtained as:

$$
N_{3}(t)=N_{03}\left[\exp \int\left(a_{3}-a_{33} N_{3}\right)\right] d t
$$

The exponential function is always non-negative and the initial population $N_{03}$ is assumed to be positive. Therefore, $N_{3}(t)>0$ for allI $\geq 0$. Hence, all the solutions of the system (4-6) are positive for all $t \geq 0$ under the considered positive initial conditions.

b) Boundedness of $N_{1}(t), N_{2}(t)$ and $N_{3}(\mathrm{t})$

In this section, all the solutions of system (4), (5) and (6) are shown to be bounded. The boundedness of the system is presented in the form of a proposition as follows:

Proposition 2: All solutions of the system (4), (5) and (6) with positive initial conditions are bounded.

Proof: Boundedness of the third population: To show that the population is bounded it is appropriate to start with the third equation from the model system.

Thus,

$$
\frac{d N_{3}}{d t}=a_{3} N_{3}-a_{33} N^{2}{ }_{3}
$$

Using partial fractions and performing of some simple algebraic manipulations reduces the equation to

$$
\left[\frac{a_{33}}{1-a_{33} N_{3}}+\frac{1}{a_{3} N_{3}}\right] N_{3}=d t
$$

Application of integration reduces it to: $\log \left[\frac{a_{3} N_{3}}{1-a_{33} N_{3}}\right]=$ $t+\log k$

Here the quantity is an arbitrarily integral constant and must be positive. Applications of anti-logarithm lead to: $\left[\frac{a_{3} N_{3}}{1-a_{33} N_{3}}\right]=k e^{t}$

Equivalently; $N_{3}\left(a_{3}+a_{33} k e^{t}\right)=k e^{t}$

$N_{3}=\frac{k e^{t}}{a_{3}+a_{33} k e^{t}}$, this implies that $N_{3}=\frac{k}{a_{3} e^{-t}+a_{33} k}$.

Now $e^{-t} \rightarrow 0$ as $\mathrm{t} \rightarrow \infty$. Thus, the system for $N_{3}$ takes the form as: $N_{3}=\frac{1}{a_{33}} \leq a_{33}$

Therefore the third population $N_{3}$ is bounded above by inverse of its self inhibition coefficient. That is the host population is bounded above by its carrying capacity.

Boundedness of the first population: To show that the first population is bounded it is appropriate to start with the equation (4) from the model system.

$$
\frac{d N_{1}}{d t}=a_{1} N_{1}-a_{11} N^{2}{ }_{1}+\frac{a N_{1} N_{3}}{1+a h N_{1}}-a_{12} N_{1} N_{2}
$$

It is true that the term $\left(a_{12} N_{1} N_{2}\right)$ and $\left(a_{11} N_{1}{ }_{1}\right)$ are positive since each member of it is a positive quantity. Thus, without loss of generality

$$
\frac{d N_{1}}{d t} \leq a_{1} N_{1}+\frac{a N_{1} N_{3}}{1+a h N_{1}}
$$

$\left(\frac{1}{N_{1}}\right) \frac{d N_{1}}{d t} \leq a_{1}+\frac{a_{1} N_{3}}{1+a h N_{1}} \leq a_{1}+\frac{a a_{33}}{1+a h N_{1}} \leq \frac{a_{33}}{h}$, since $\frac{1}{1+a h N_{1}} \leq 1$

Thus, the inequality can be re-expressed as;

$$
\left(\frac{1}{N_{1}}\right) \frac{d N_{1}}{d t} \leq a_{1}+\frac{a_{33}}{h}
$$

On applying integration, it can be obtained that

$$
\log N_{1} \leq\left[a_{1}+\frac{a_{33}}{h}\right] t+\log m
$$

This is equivalent to $N_{1} \leq m e^{\left[a_{1}+\frac{a_{33}}{h}\right] t}$

Here the quantity $m$ is an arbitrarily integral constant and must be strictly positive due to logarithmic function. Now there arise three cases, namely the exponent may be negative or positive or zero. These cases as analyzed as follows:

i. If $\left[a_{1}+\frac{a_{33}}{h}\right]<0$ then as $\mathrm{t} \rightarrow \infty$ the exponential term takes a value zero and thus to get $N_{1} \leq 0$. But this, having negative population, is biologically not feasible. Hence this possibility is not considered.

ii. If $\left[a_{1}+\frac{a_{33}}{h}\right]>0$ then as $\mathrm{t} \rightarrow \infty$ the exponential term takes a value $\infty$ and thus to get $N_{1} \leq \infty$. But this, having $\infty$ population, is biologically not feasible. It is an unbounded case. Hence this possibility is not considered.

iii. If $\left[a_{1}+\frac{a_{33}}{h}\right]=0$ then as $\mathrm{t} \rightarrow \infty$ the exponential term takes a value 1 and thus reduces to $N_{1} \leq \mathrm{m}$. Hence this possibility is considered. Thus the population $N_{1}$ is bounded above by arbitrarily positive constant $\mathrm{m}$. In other word the death rate more prominent than the other parameters that is why this possibility is happen.

Boundedness of the second population: To show that the population is bounded it is appropriate to start with the equation (5) from the model system.

Thus,

$$
\begin{gathered}
\frac{d N_{2}}{d t}=a_{2} N_{2}-a_{22} N_{2}^{2}+a_{21} N_{1} N_{2}-a_{23} N_{2} N_{3} \\
\frac{d N_{2}}{d t}=N_{2}\left[a_{2}-a_{22} N_{2}+a_{21} N_{1}-a_{23} N_{3}\right]
\end{gathered}
$$

It has been already shown that the population sizes of the two populations are bounded. That is, $N_{3} \leq a_{33}$ and $N_{1} \leq \mathrm{m}$. Here it can be observed that $a_{21} N_{1} \leq a_{21} m$ and $a_{23} N_{3} \leq$ $a_{23} a_{33}$.

In view of these observations the equation takes the form as:

$$
\begin{gathered}
\frac{d N_{2}}{d t} \leq N_{2}\left[a_{2}-a_{22} N_{2}+a_{21} m-a_{23} a_{33}\right] \\
\frac{d N_{2}}{d t} \leq N_{2}\left[n-a_{22} N_{2}\right] . \text { Here } n=\left[a_{2}+a_{21} m-a_{23} a_{33}\right] .
\end{gathered}
$$

Applying partial fraction and integrating:

$$
\ln \left[\frac{N_{2}}{n-a_{22} N_{2}}\right] \leq n t+\ln p
$$


Here the quantity $\mathrm{p}$ is an arbitrarily integral constant and must be strictly positive due to logarithmic function.

$$
\begin{gathered}
{\left[\frac{N_{2}}{n-a_{22} N_{2}}\right] \leq p e^{n t}} \\
N_{2} \leq \frac{p n e^{n t}}{1+a_{22} p e^{n t}} \\
N_{2} \leq \frac{p n}{e^{-n t}+a_{22} p}
\end{gathered}
$$

It can be observed that $e^{-n t} \rightarrow 0$ as $\mathrm{t} \rightarrow \infty$ and thus $N_{2} \leq \frac{n}{a_{22}}$

That is, the second population is bounded above by $N_{2} \leq \frac{\left[a_{2}+a_{21} m-a_{23} a_{33}\right]}{a_{22}}$.

Therefore, the solution of the model system (4-6) is bounded.

\section{The Steady States}

The critical points of the system can be obtained by setting $\frac{N i(t}{d t}=0, \mathrm{i}=1,2,3$. in the model equations. This leads to the following optional relations;

$$
\begin{gathered}
N_{1}=0 \\
\text { Or, }\left[a_{1}-a_{11} N_{1}+\frac{a}{1+a h N_{1}} N_{3}-a_{12} N_{2}\right]=0 \\
N_{2}=0 \\
\text { Or, }\left[a_{2}-a_{22} N_{2}+a_{21} N_{1}-a_{23} N_{3}\right]=0 \\
N_{3}=0 \\
\text { Or, }\left[a_{3}-a_{33} N_{3}\right]=0
\end{gathered}
$$

The solutions of these optional relations can be the equilibrium points. There are eight possible combinations of the relations. These combinations and their solutions or equilibrium points are as mentioned below:

Table 2. Possible combinations of the solution of the systems.

\begin{tabular}{ll}
\hline Combination of relations & Equilibrium points \\
\hline$(2.4)(2.6)(2.8)$ & $E_{0}$ \\
$(2.4)(2.6)(2.9)$ & $E_{1}$ \\
$(2.4)(2.7)(2.8)$ & $E_{2}$ \\
$(2.5)(2.6)(2.8)$ & $E_{3}$ \\
$(2.4)(2.7)(2.9)$ & $E_{4}$ \\
$(2.5)(2.6)(2.9)$ & $E_{5}$ \\
$(2.5)(2.7)(2.8)$ & $E_{6}$ \\
$(2.5)(2.7)(2.9)$ & $E_{7}$ \\
$(2.4)(2.5)(2.9)$ & Not biologically feasible \\
\hline
\end{tabular}

It can be observed that the first two combinations lead to the same equilibrium point while the last combination leads to biologically infeasible solution. Thus, eight equilibrium points are possible. Now the coordinates of these possible equilibrium points as given below:

(1) Fully washed out state: $E_{0}(0,0,0)$, i.e., N1 $=0, \mathrm{~N} 2=0$, $\mathrm{N} 3=0$. The extinction of all populations equilibrium $\left(E_{0}\right)$ always exists.

(2) States in which two species are washed out: $E_{1}\left(0,0, \frac{\mathrm{a}_{3}}{\mathrm{a}_{33}}\right), E_{2}\left(0, \frac{\mathrm{a}_{2}}{\mathrm{a}_{22}}, 0\right), E_{3}\left(\frac{\mathrm{a}_{1}}{\mathrm{a}_{11}}, 0,0\right)$

(3) States in which one species is washed out: $E_{4}\left(0, \mathrm{~N}_{2}^{*}, \frac{\mathrm{a}_{3}}{\mathrm{a}_{33}}\right), E_{5}\left(\mathrm{~N}_{1}^{*}, 0, \frac{\mathrm{a}_{3}}{\mathrm{a}_{33}}\right), E_{6}\left(\mathrm{~N}_{1}^{* *}, \mathrm{~N}_{2}^{* *}, 0\right)$

Where $\mathrm{N}_{1}^{*}=\frac{-\mathrm{k}+\sqrt{\mathrm{k}^{2}-4 \mathrm{M}}}{2}, k=\frac{a_{1}}{\mathrm{a}_{22} \mathrm{a}_{33}}-\frac{1}{a h} ; M=\frac{a_{1}+a_{3} a}{\mathrm{a}_{22} \mathrm{a}_{33} \mathrm{ah}}$; $\mathrm{N}_{1}^{* *}=\frac{\mathrm{a}_{1} \mathrm{a}_{22}-\mathrm{a}_{2} \mathrm{a}_{12}}{\mathrm{a}_{11} \mathrm{a}_{22}+\mathrm{a}_{12} \mathrm{a}_{21}}$

$$
\begin{gathered}
\mathrm{N}_{2}^{*}=\frac{a_{2}}{\mathrm{a}_{22}}-\frac{a_{3} \mathrm{a}_{23}}{\mathrm{a}_{22} \mathrm{a}_{33}} \text { when } \mathrm{a}_{2} \mathrm{a}_{33}>a_{3} \mathrm{a}_{23} ; \mathrm{N}_{2}^{* *}=\frac{a_{1}}{\mathrm{a}_{12}}- \\
\frac{a_{11}}{\mathrm{a}_{12}}\left[\frac{\mathrm{a}_{1} \mathrm{a}_{22}-\mathrm{a}_{2} \mathrm{a}_{12}}{\mathrm{a}_{11} \mathrm{a}_{22}+\mathrm{a}_{12} \mathrm{a}_{21}}\right] .
\end{gathered}
$$

(4) States in which the three species exists: $E_{7}\left(\mathrm{~N}_{1}^{* * *}, \mathrm{~N}_{2}^{* * *}, \mathrm{~N}_{3}^{* * *}\right)$, where

$$
\begin{gathered}
\mathrm{N}_{1}^{* * *}=\frac{-\mathrm{P}-\sqrt{\mathrm{P}^{2}-4 Q S}}{2 \mathrm{Q}} ; \mathrm{Q}=-\left[\mathrm{a}_{11} \mathrm{ah}+\frac{\mathrm{a}_{21} \mathrm{a}_{12} \mathrm{ah}}{\mathrm{a}_{22}}\right], \\
\mathrm{P}=\mathrm{a}_{1} \mathrm{ah}+\frac{\mathrm{a}_{3} \mathrm{a}_{12} \mathrm{a}_{23} \mathrm{ah}}{\mathrm{a}_{22} \mathrm{a}_{33}}-\left[\frac{\mathrm{a}_{22} \mathrm{a}_{11}+\mathrm{a}_{12} \mathrm{a}_{2} a h+\mathrm{a}_{21} \mathrm{a}_{12}}{\mathrm{a}_{22}}\right], \\
S=\mathrm{a}_{1}+\frac{\mathrm{a}_{3} \mathrm{a}}{\mathrm{a}_{33}}-\frac{\mathrm{a}_{12} \mathrm{a}_{2}}{\mathrm{a}_{22}}+\frac{\mathrm{a}_{3} \mathrm{a}_{23} \mathrm{a}_{12}}{\mathrm{a}_{22} \mathrm{a}_{33}} . \\
\mathrm{N}_{2}^{* * *}=\frac{\mathrm{a}_{2}}{\mathrm{a}_{22}}+\frac{\mathrm{a}_{21}}{\mathrm{a}_{22}} \mathrm{~N}_{1}^{* * *}-\frac{\mathrm{a}_{3} \mathrm{a}_{23}}{\mathrm{a}_{22} \mathrm{a}_{33}} ; \\
\mathrm{N}_{3}^{* * *}=\frac{\mathrm{a}_{3}}{\mathrm{a}_{33}} .
\end{gathered}
$$

\section{Stability Analysis of the Equilibrium States}

To analyze the stability near the equilibrium points the community matrix called Jacobian matrix is and the conditions for stability of the equilibrium state are determined and stated as follows.

Community matrix

$$
\text { Let } \frac{d N_{1}}{d t}=h\left(N_{1}, N_{2}, N_{3}\right), \frac{d N_{2}}{d t}=f\left(N_{1}, N_{2}, N_{3}\right) \text {, and } \frac{d N_{3}}{d t}=g
$$$$
\left(N_{1}, N_{2}, N_{3}\right) \text {. }
$$

Where the functions are given as; $h=a_{1} N_{1}-a_{11} N^{2}{ }_{1}+$ $\frac{a}{1+a h N_{1}} N_{1} N_{3}-a_{12} N_{1} N_{2}$,

$f=a_{2} N_{2}-a_{22} N_{2}^{2}+a_{21} N_{1} N_{2}-a_{23} N_{2} N_{3}$ and $g=$ $a_{3} N_{3}-a_{33} N_{3}^{2}$.

Moreover, the components of the Jacobian matrix are given by; 


$$
J=\left[\begin{array}{lll}
\frac{\partial h}{\partial N_{1}} & \frac{\partial h}{\partial N_{2}} & \frac{\partial h}{\partial N_{3}} \\
\frac{\partial f}{\partial N_{1}} & \frac{\partial f}{\partial N_{2}} & \frac{\partial f}{\partial N_{3}} \\
\frac{\partial g}{\partial N_{1}} & \frac{\partial g}{\partial N_{2}} & \frac{\partial g}{\partial N_{3}}
\end{array}\right]
$$

Therefore, the Jacobian matrix takes the components as;

$$
J=\left[\begin{array}{ccc}
a_{1}-2 a_{11} N_{1}+\frac{a N_{3}}{\left(1+a h N_{1}\right)^{2}}-a_{12} N_{2} & -a_{12} N_{1} & \frac{a N_{1}}{1+a h N_{1}} \\
a_{21} N_{2} & a_{2}-2 a_{22} N_{2}+a_{21} N_{1}-a_{23} N_{3} & -a_{23} N_{2} \\
0 & 0 & a_{3}-2 a_{33} N_{3}
\end{array}\right]
$$

Analysis of the equilibrium points

\subsection{Local Stability of the Steady State}

In this section, the stability of the model (2.1), (2.2) and (2.3) at the equilibrium point is analyzed. The local stability of the steady state is determined based on the nature of the eigenvalues of the variation matrix.

Theorem 1: The trivial equilibrium point $E_{0}(0,0,0)$ is unstable.

Proof: The eigenvalues of the variation matrix $\left(\mathrm{J}_{0}\right)$ at $E_{0}$ are given by;

$\operatorname{det}\left(\left(\mathrm{J}_{0}-\lambda I\right)=0\right.$. Hence, $\lambda_{1}=a_{1}, \lambda_{2}=a_{2}, \lambda_{3}=a_{3}$. Since $a_{1}, a_{2}, a_{3}>0$. Thus, the washed out state $E_{0}$ is unstable.

Theorem 2: States in which two species are washed out are unstable, i. e, $E_{1}, E_{2}$ and $E_{3}$.

Proof: The characteristic equations at $E_{1}$ is;

$$
\left[a_{1}+\frac{a a_{3}}{a_{33}}-\lambda\right]\left[a_{2}-\frac{a_{23} a_{3}}{a_{33}}-\lambda\right]\left[-a_{3}-\lambda\right]=0
$$

The eigenvalues of this equation becomes; $\lambda_{1}=a_{1}+$ $\frac{a a_{3}}{a_{33}}, \lambda_{2}=a_{2}-\frac{a_{23} a_{3}}{a_{33}}, \lambda_{3}=-a_{3}$.

Since $\lambda_{1}>0$ unconditional positive. Hence, the steady state $E_{1}$ is unstable.

The characteristic equations at $E_{2}$ is solved as;

$$
\left[a-\frac{a_{12} a_{2}}{a_{22}}-\lambda\right]\left[-a_{2}-\lambda\right]\left[a_{3}-\lambda\right]=0
$$

The eigenvalues of this equation becomes; $\lambda_{1}=a-$ $\frac{a_{12} a_{2}}{a_{22}}, \lambda_{2}=-a_{2}, \lambda_{3}=a_{3}$.

Since $\lambda_{3}>0$ unconditional positive. Hence, the steady state $E_{2}$ is unstable.

The characteristic equations at $E_{2}$ is solved as;

$$
\left[-a_{1}-\lambda\right]\left[a_{2}+\frac{a_{21} a_{1}}{a_{11}}-\lambda\right]\left[a_{3}-\lambda\right]=0
$$

The eigenvalues of this equation becomes; $\lambda_{1}=-a_{1}, \lambda_{2}=$ $a_{2}+\frac{a_{21} a_{1}}{a_{11}}, \lambda_{3}=a_{3}$.
As $\lambda_{3}>0$ unconditional positive. Hence, the equilibrium point $E_{3}$ is unstable.

Therefore, the states in which the two species washed out are unstable.

Theorem 3: States in which one species is washed out.

i. The steady states $E_{4}, E_{5}$ are stable under the following conditions.

That is $E_{4}$ is stable when $m<n$, where $m=a_{1}+$ $a_{22} a_{33}+a a_{3} a_{22}+a_{12} a_{3} a_{23}$,

$n=a_{12} a_{2} a_{33}$ and $a_{3} a_{23}<a_{2} a_{33}$ a nd $E_{5}$ is stable when $a_{1}<2 a_{11} \mathrm{~N}_{1}^{*}+\left(\frac{a a_{3}}{a_{33}}\right)\left[\frac{1}{\left(1+a h N_{1}^{*}\right)^{2}}\right]$ and $a_{2}+a_{21} \mathrm{~N}_{1}^{*}<$ $\frac{a_{3} a_{23}}{a_{33}}$, where $N_{1}^{*}=\frac{\left(\frac{1}{a h}-\frac{a_{1}}{a_{22} a_{33}}\right)+\sqrt{\left(\frac{a_{22} a_{33}-a a_{1} a_{1}}{\text { aha }_{22} a_{33}}\right)^{2}-4\left(\frac{a_{1}+a_{3}}{a_{h a} a_{22} a_{33}}\right)}}{2}$

Proof: The Jacobian matrix (2.10) is evaluated at $E_{4}$ with the following eigenvalues:

$$
J=\left(\begin{array}{ccc}
a_{1}+\frac{a a_{3}}{a_{33}}-a_{12} \mathrm{~N}_{2}^{*} & 0 & 0 \\
a_{21} \mathrm{~N}_{2}^{*} & a_{2}-2 a_{22} \mathrm{~N}_{2}^{*}-a_{23} \mathrm{~N}_{3}^{*} & -a_{23} \mathrm{~N}_{2}^{*} \\
0 & 0 & a_{3}-2 a_{33} \mathrm{~N}_{3}^{*}
\end{array}\right)
$$

The eigenvalues of $J\left(E_{4}\right)$ are obtained by solving the characteristic equation;

$$
\begin{gathered}
{\left[a_{1}+\frac{a a_{3}}{a_{33}}-a_{12} \mathrm{~N}_{2}^{*}-\lambda\right]\left[a_{2}-2 a_{22} \mathrm{~N}_{2}^{*}-a_{23} \mathrm{~N}_{3}^{*}-\lambda\right]\left[a_{3}-\right.} \\
\left.2 a_{33} \mathrm{~N}_{3}^{*}-\lambda\right]=0, \text { where, } \\
\mathrm{N}_{2}^{*}=\frac{a_{2}}{\mathrm{a}_{22}}-\frac{a_{3} \mathrm{a}_{23}}{\mathrm{a}_{22} \mathrm{a}_{33}}, \mathrm{~N}_{3}^{*}=\frac{\mathrm{a}_{3}}{\mathrm{a}_{33}} .
\end{gathered}
$$

Thus the eigenvalues are $\lambda_{1}=\frac{m-n}{a_{22} \mathrm{a}_{3}}, \lambda_{2}=\frac{a_{3} \mathrm{a}_{23}}{\mathrm{a}_{33}}-$ $a_{2}, \lambda_{3}=-a_{3}$

Here, $\lambda_{3}<0$, conditional negative but $\lambda_{1}<0$ when $m<n$ and $\lambda_{2}<0$ when $a_{3} a_{23}<a_{2} a_{33}$.

Therefore, $E_{4}$ is stable under the set criteria, otherwise unstable.

Similarly, we can show $E_{5}$ as follows;

The evaluation of Jacobian matrix (2.10) at $E_{5}$ gives 


$$
J\left(E_{5}\right)=\left(\begin{array}{ccc}
a_{1}-2 a_{11} \mathrm{~N}_{1}^{*}+\frac{a \mathrm{~N}_{3}^{*}}{\left(1+a h \mathrm{~N}_{1}^{*}\right)^{2}} & -a_{12} \mathrm{~N}_{1}^{*} & \frac{a \mathrm{~N}_{1}^{*}}{1+a h \mathrm{~N}_{1}^{*}} \\
0 & a_{2}+a_{21} \mathrm{~N}_{1}^{*}-a_{23} \mathrm{~N}_{3}^{*} & 0 \\
0 & 0 & a_{3}-2 a_{33} \mathrm{~N}_{3}^{*}
\end{array}\right)
$$

The characteristics equation of $J\left(E_{5}\right)$ is given by the following equation:

$$
\begin{gathered}
{\left[a_{1}-2 a_{11} \mathrm{~N}_{1}^{*}+\frac{a \mathrm{~N}_{3}^{*}}{\left(1+a h \mathrm{~N}_{1}^{*}\right)^{2}}-\lambda\right]} \\
{\left[a_{2}+a_{21} \mathrm{~N}_{1}^{*}-a_{23} \mathrm{~N}_{3}^{*}-\lambda\right]\left[a_{3}-2 a_{33} \mathrm{~N}_{3}^{*}-\lambda\right]=0}
\end{gathered}
$$

This gives, $\lambda_{1}=a_{1}-2 a_{11} \mathrm{~N}_{1}^{*}+\frac{a \mathrm{~N}_{3}^{*}}{\left(1+a h \mathrm{~N}_{1}^{*}\right)^{2}}, \lambda_{2}=a_{2}+$ $a_{21} \mathrm{~N}_{1}^{*}-a_{23} \mathrm{~N}_{3}^{*}, \lambda_{3}=a_{3}-2 a_{33} \mathrm{~N}_{3}^{*}$.

Where

$$
\mathrm{N}_{1}^{*}=\frac{\left(\frac{1}{a h}-\frac{a_{1}}{a_{22} a_{33}}\right)+\sqrt{\left(\frac{a_{22} a_{33}-a h a_{1}}{a h a 22_{23} a_{33}}\right)^{2}-4\left(\frac{a_{1}+a_{3}}{a h a_{22} a_{33}}\right)}}{2} ;
$$

$\mathrm{N}_{3}^{*}=\frac{\mathrm{a}_{3}}{\mathrm{a}_{33}}$

Here, $\lambda_{3}<0$ conditional negative and $\lambda_{2}<0$ when $a_{2}+a_{21} \mathrm{~N}_{1}^{*}<\frac{a_{3} \mathrm{a}_{23}}{\mathrm{a}_{33}}$ but $\lambda_{1}<0$ when the following conditions are carefully holds. That is, $a_{1}<2 a_{11} \mathrm{~N}_{1}^{*}+$ $\frac{a \mathrm{~N}_{3}^{*}}{\left(1+a h \mathrm{~N}_{1}^{*}\right)^{2}}$ since $\mathrm{N}_{1}^{*}$ is positive quantity.

Hence, $E_{5}$ is locally stable under the set conditions, otherwise unstable.

ii. The steady state $E_{6}$ is unstable.

Proof: The evaluation of Jacobian matrix (2.10) at $E_{6}$ gives

$$
J\left(E_{6}\right)=\left(\begin{array}{ccc}
a_{1}-2 a_{11} \mathrm{~N}_{1}^{* *}-a_{12} \mathrm{~N}_{2}^{* *} & -a_{12} \mathrm{~N}_{1}^{*} & \frac{a \mathrm{~N}_{1}^{* *}}{1+a h \mathrm{~N}_{1}^{* *}} \\
a_{21} \mathrm{~N}_{2}^{* *} & a_{2}-2 a_{22} \mathrm{~N}_{2}^{* *}+a_{21} \mathrm{~N}_{1}^{* *} & -a_{23} \mathrm{~N}_{2}^{* *} \\
0 & 0 & a_{3}
\end{array}\right)
$$

The characteristics equation of $J\left(E_{6}\right)$ is given by the following equation:

$$
J=\left(\begin{array}{c}
a_{1}-2 a_{11} \mathrm{~N}_{1}^{* * *}+\frac{a \mathrm{~N}_{3}^{* * *}}{\left(1+a h \mathrm{~N}_{1}^{* * *}\right)^{2}}-a_{12} \mathrm{~N}_{2}^{* * *} \\
a_{21} \mathrm{~N}_{2}^{* * *} \\
0
\end{array}\right.
$$

The characteristics equation of $J\left(E_{7}\right)$ is given by the following quadratic equation

$$
\begin{gathered}
{\left[a_{1}-2 a_{11} \mathrm{~N}_{1}^{* * *}+\frac{a \mathrm{~N}_{3}^{* * *}}{\left(1+a h \mathrm{~N}_{1}^{* * *}\right)^{2}}-a_{12} \mathrm{~N}_{2}^{* * *}-\lambda\right]} \\
{\left[a_{2}-2 a_{22} \mathrm{~N}_{2}^{* * *}\right.} \\
\left.+a_{21} \mathrm{~N}_{1}^{* * *}-a_{23} \mathrm{~N}_{3}^{* * *}-\lambda\right]\left[a_{3}-2 a_{33} \mathrm{~N}_{3}^{* * *}\right. \\
-\lambda] \\
+\left(a_{12} \mathrm{~N}_{1}^{* * *}\right)\left(a_{21} \mathrm{~N}_{2}^{* * *}\right)\left(a_{3}-2 a_{33} \mathrm{~N}_{3}^{* * *}-\lambda\right) \\
=0
\end{gathered}
$$

This implies,

$$
\begin{gathered}
{\left[a_{1}-2 a_{11} \mathrm{~N}_{1}^{* * *}+\frac{a \mathrm{~N}_{3}^{* * *}}{\left(1+a h \mathrm{~N}_{1}^{* * *}\right)^{2}}-a_{12} \mathrm{~N}_{2}^{* * *}-\lambda\right]\left[a_{2}-\right.} \\
\left.2 a_{22} \mathrm{~N}_{2}^{* * *}+a_{21} \mathrm{~N}_{1}^{* * *}-a_{23} \mathrm{~N}_{3}^{* * *}-\lambda\right]\left[a_{3}-2 a_{33} \mathrm{~N}_{3}^{* * *}-\lambda\right]+ \\
\left(a_{12} \mathrm{~N}_{1}^{* * *}\right)\left(a_{21} \mathrm{~N}_{2}^{* * *}\right)=0 ;\left(a_{3}-2 a_{33} \mathrm{~N}_{3}^{* * *}-\lambda\right)=0
\end{gathered}
$$

$$
\begin{gathered}
{\left[a_{1}-2 a_{11} \mathrm{~N}_{1}^{* *}-a_{12} \mathrm{~N}_{2}^{* *}-\lambda\right]\left[a_{2}-2 a_{22} \mathrm{~N}_{2}^{* *}+a_{21} \mathrm{~N}_{1}^{* *}-\lambda\right]} \\
{\left[a_{3}-\lambda\right]+\left[a_{12} \mathrm{~N}_{1}^{*}\right]\left[a_{21} \mathrm{~N}_{2}^{* *}\right]\left[a_{3}-\lambda\right]=0}
\end{gathered}
$$

This gives,

$$
\begin{gathered}
{\left[a_{1}-2 a_{11} \mathrm{~N}_{1}^{* *}-a_{12} \mathrm{~N}_{2}^{* *}-\lambda\right]\left[a_{2}-2 a_{22} \mathrm{~N}_{2}^{* *}+a_{21} \mathrm{~N}_{1}^{* *}-\lambda\right]} \\
+\left[a_{12} \mathrm{~N}_{1}^{*}\right]\left[a_{21} \mathrm{~N}_{2}^{* *}\right]=0,\left[a_{3}-\lambda\right]=0
\end{gathered}
$$

Here, $\lambda_{3}>0$ un conditional positive. Thus $E_{6}$ is unstable whatever the case is.

Theorem 4: States in which the three species exists: $E_{7}\left(\mathrm{~N}_{1}^{* * *}, \mathrm{~N}_{2}^{* * *}, \mathrm{~N}_{3}^{* * *}\right)$, is where

$$
\begin{gathered}
\mathrm{N}_{1}^{* * *}=\frac{-\mathrm{P}-\sqrt{\mathrm{P}^{2}-4 Q S}}{2 \mathrm{Q}} ; \mathrm{Q}=-\left[\mathrm{a}_{11} \mathrm{ah}+\frac{\mathrm{a}_{21} \mathrm{a}_{12} \mathrm{ah}}{\mathrm{a}_{22}}\right], \\
\mathrm{P}=\mathrm{a}_{1} \mathrm{ah}+\frac{\mathrm{a}_{3} \mathrm{a}_{12} \mathrm{a}_{23} \mathrm{ah}}{\mathrm{a}_{22} \mathrm{a}_{33}}-\left[\frac{\mathrm{a}_{22} \mathrm{a}_{11}+\mathrm{a}_{12} \mathrm{a}_{2} a h+\mathrm{a}_{21} \mathrm{a}_{12}}{\mathrm{a}_{22}}\right], \\
S=\mathrm{a}_{1}+\frac{\mathrm{a}_{3} \mathrm{a}}{\mathrm{a}_{33}}-\frac{\mathrm{a}_{12} \mathrm{a}_{2}}{\mathrm{a}_{22}}+\frac{\mathrm{a}_{3} \mathrm{a}_{23} \mathrm{a}_{12}}{\mathrm{a}_{22} \mathrm{a}_{33}} . \\
\mathrm{N}_{2}^{* * *}=\frac{\mathrm{a}_{2}}{\mathrm{a}_{22}}+\frac{\mathrm{a}_{21}}{\mathrm{a}_{22}} \mathrm{~N}_{1}^{* * *}-\frac{\mathrm{a}_{3} \mathrm{a}_{23}}{\mathrm{a}_{22} \mathrm{a}_{33}} ; \\
\mathrm{N}_{3}^{* * *}=\frac{\mathrm{a}_{3}}{\mathrm{a}_{33}} .
\end{gathered}
$$

The Jacobian matrix at an equilibrium point $E_{7}=$ $\left(\mathrm{N}_{1}^{* * *}, \mathrm{~N}_{2}^{* * *}, \mathrm{~N}_{3}^{* * *}\right)$ is given by;

$$
\left.\begin{array}{cc}
-a_{12} \mathrm{~N}_{1}^{* * *} & \frac{a \mathrm{~N}_{1}^{* * *}}{1+a h \mathrm{~N}_{1}^{* * *}} \\
a_{2}-2 a_{22} \mathrm{~N}_{2}^{* * *}+a_{21} \mathrm{~N}_{1}^{* * *}-a_{23} \mathrm{~N}_{3}^{* * *} & -a_{23} \mathrm{~N}_{2}^{* * *} \\
0 & a_{3}-2 a_{33} \mathrm{~N}_{3}^{* * *}
\end{array}\right)
$$

Where $A=2 a_{11} \mathrm{~N}_{1}^{* * *}-\frac{a \mathrm{~N}_{3}^{* * *}}{\left(1+a h \mathrm{~N}_{1}^{* *}\right)^{2}}+a_{12} \mathrm{~N}_{2}^{* * *}-a_{1}, B=$

$$
\begin{gathered}
2 a_{22} \mathrm{~N}_{2}^{* * *}-a_{21} \mathrm{~N}_{1}^{* * *}+a_{23} \mathrm{~N}_{3}^{* * *}-a_{2}, \\
D=A B+\left(a_{12} \mathrm{~N}_{1}^{* * *}\right)\left(a_{21} \mathrm{~N}_{2}^{* * *}\right)
\end{gathered}
$$

From the Routh-Hurwitz criterion, we can conclude that $H=1>0,(A+B)>0$ or $D>0$

Hence, $E_{7}$ is locally asymptotically stable.

\subsection{Global Stability of Steady State}

The main goal of this model formulation is targeted to eradicate the enemy. Mathematically, this can be achieved whenever the second species free equilibrium is stable. The sufficient condition for this equilibrium to be globally 
asymptotically stable is given by the following theorem.

Theorem 5: If $a_{2}+a_{21} \mathrm{~N}_{1}^{*}<\frac{a_{3} \mathrm{a}_{23}}{\mathrm{a}_{33}}$ and $a_{1}<2 a_{11} \mathrm{~N}_{1}^{*}+$ $\left(\frac{a a_{3}}{a_{33}}\right)\left(\frac{1}{\left(1+a h N_{1}^{*}\right)^{2}}\right)$ the steady states $E_{5}$ is globally asymptotically stable.

Proof: Consider the Lyapunov function derived from the integral form;

$$
\int_{x^{*}}^{x} \frac{u-x^{*}}{u} d u
$$

Now, let $\quad V\left(N_{1}, N_{2}, N_{3}\right)=N_{1}-\mathrm{N}_{1}^{*}-\mathrm{N}_{1}^{*} \ln \frac{N_{1}}{\mathrm{~N}_{1}^{*}}+$ $l\left(N_{3}-\mathrm{N}_{3}^{*}-\mathrm{N}_{3}^{*} \ln \frac{N_{3}}{\mathrm{~N}_{3}^{*}}\right)$, where 1 is some positive constant assumed.

Now, the differential of $v$ with respect to $t$ and after some algebraic manipulations reduces to the following form:

$$
\begin{gathered}
\dot{V}\left(N_{1}, N_{2}, N_{3}\right)=\left[\frac{N_{1}-\mathrm{N}_{1}^{*}}{N_{1}}\right]\left(\frac{d N_{1}}{d t}\right)+l\left[\frac{N_{3}-\mathrm{N}_{3}^{*}}{N_{3}}\right]\left(\frac{d N_{3}}{d t}\right) \\
=\left[\frac{N_{1}-\mathrm{N}_{1}^{*}}{N_{1}}\right]\left[a_{1} N_{1}-a_{11} N_{1}^{2}+\frac{a}{1+a h N_{1}} N_{1} N_{3}-a_{12} N_{1} N_{2}\right] \\
+l\left[\frac{N_{3}-\mathrm{N}_{3}^{*}}{N_{3}}\right]\left[a_{3} N_{3}-a_{33} N_{3}^{2}\right]
\end{gathered}
$$

$$
\begin{gathered}
=\left[N_{1}-\mathrm{N}_{1}^{*}\right]\left[a_{1}-a_{11} N_{1}+\frac{a N_{3}}{1+a h N_{1}}\right] \\
+l\left[N_{3}-\mathrm{N}_{3}^{*}\right]\left[a_{3}-a_{33} N_{3}\right] \\
=-\left[N_{1}-\mathrm{N}_{1}^{*}\right]^{2}\left(\frac{a_{1}}{a_{11}}-\omega\right)+-l\left[N_{3}-\mathrm{N}_{3}^{*}\right]^{2}\left[\frac{a_{3}}{a_{33}}\right] \\
=-\left[\left(N_{1}-\mathrm{N}_{1}^{*}\right)^{2}\left(\frac{a_{1}}{a_{11}}-\omega\right)+l\left[N_{3}-\mathrm{N}_{3}^{*}\right]^{2}\left(\frac{a_{3}}{a_{33}}\right)\right]
\end{gathered}
$$

Choosing, $l=\frac{a_{33}}{a_{3}}$ and letting $\omega=\frac{a N_{3}}{1+a h N_{1}}$ for the sake of simplicity.

Thus, $\frac{d V}{d t}<0$, i.e., $v$ is positive definite and also $\dot{V}\left(\mathrm{~N}_{1}^{*}, \mathrm{~N}_{2}^{*}, \mathrm{~N}_{3}^{*}\right)=0$. Therefore $E_{5}$ is globally asymptotically stable.

\begin{tabular}{|c|c|c|c|c|c|c|c|c|c|c|c|c|}
\hline \multirow{2}{*}{ Figures } & \multicolumn{11}{|c|}{ Estimated Parameters and their values } & \multirow[t]{2}{*}{ Remark } \\
\hline & $a_{1}$ & $a_{11}$ & $a$ & h & $a_{12}$ & $a_{2}$ & $a_{22}$ & $a_{21}$ & $a_{23}$ & $a_{3}$ & $a_{33}$ & \\
\hline 6.1 & 0.0000 & 0.0390 & 0.0000 & 0.0000 & 0.9740 & 0.0000 & 0.1430 & 0.0100 & 0.6010 & 0.0260 & 0.9740 & [16] \\
\hline 6.2 & 0.1000 & 0.0710 & 0.3000 & 0.9000 & 0.0100 & 0.6620 & 0.1170 & 0.0900 & 0.4640 & 0.9350 & 0.0650 & Assumed \\
\hline 6.3 & 0.0420 & 0.2790 & 0.1200 & 0.5100 & 0.8770 & 0.7110 & 0.5580 & 0.9450 & 0.0840 & 0.4610 & 0.2180 & Assumed \\
\hline 6.4 & 0.9350 & 0.2790 & 0.1200 & 0.5100 & 0.8770 & 0.9220 & 0.9320 & 0.9450 & 0.9220 & 0.9680 & 0.9680 & Assumed \\
\hline 6.5 & 0.3250 & 0.0260 & 0.3150 & 0.1690 & 0.4970 & 0.1200 & 0.0780 & 0.9610 & 0.9610 & 0.9450 & 0.1790 & Assumed \\
\hline
\end{tabular}

\section{Numerical Simulations and Discussion.}

To illustrate the dynamical behavior of system (2.1), (2.2), and (2.3), we perform some numerical simulations using hypothetical value of parameters.

The parameters and its values used in this study are mentioned in the following table as follow

Table 3. Parameters and their estimated values for figures (2-6).

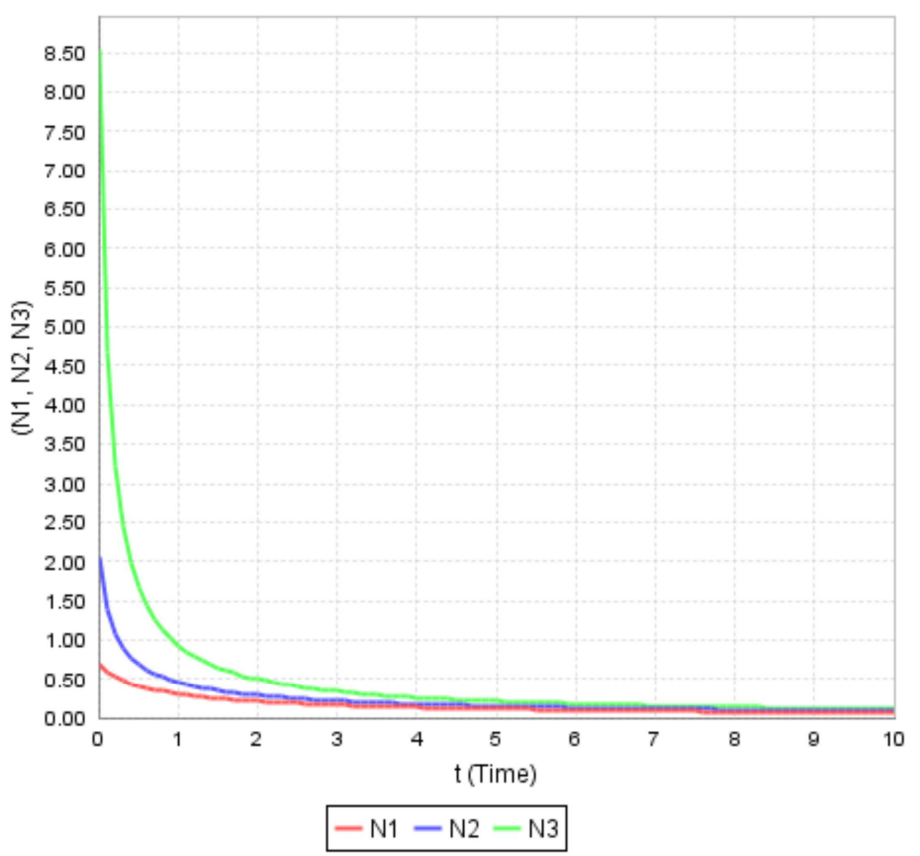

Figure 2. The dynamic behavior of the system with different initial condition and the three species washed out. 
164 Geremew Kenassa Edessa and Purnachandra Rao Koya: Modeling and Stability Analysis of a Three Species Ecosystem with the Third Species Response to the First Species in Sigmoid Functional Response Form

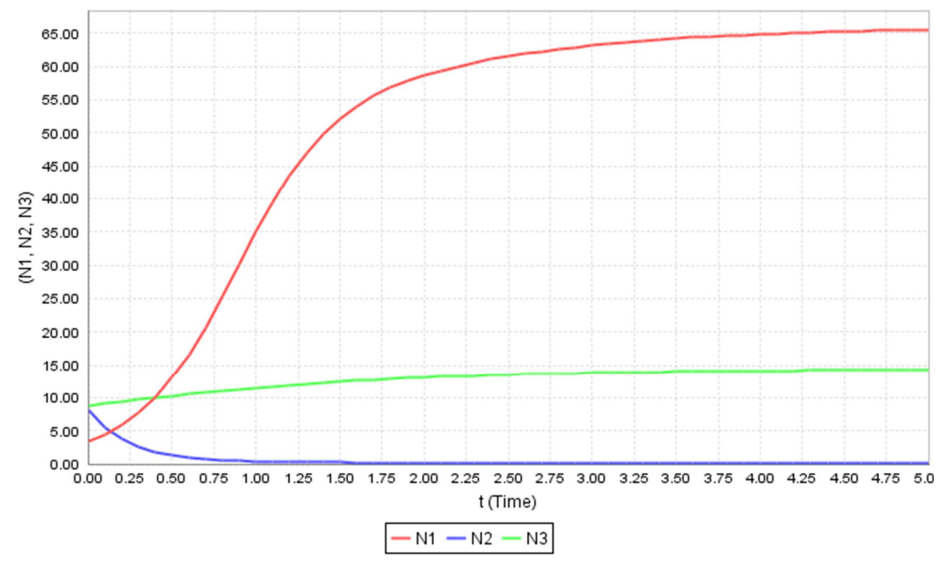

Figure 3. The dynamic behavior of the system with different initial condition and the second species washed out while the first and the third species exist.

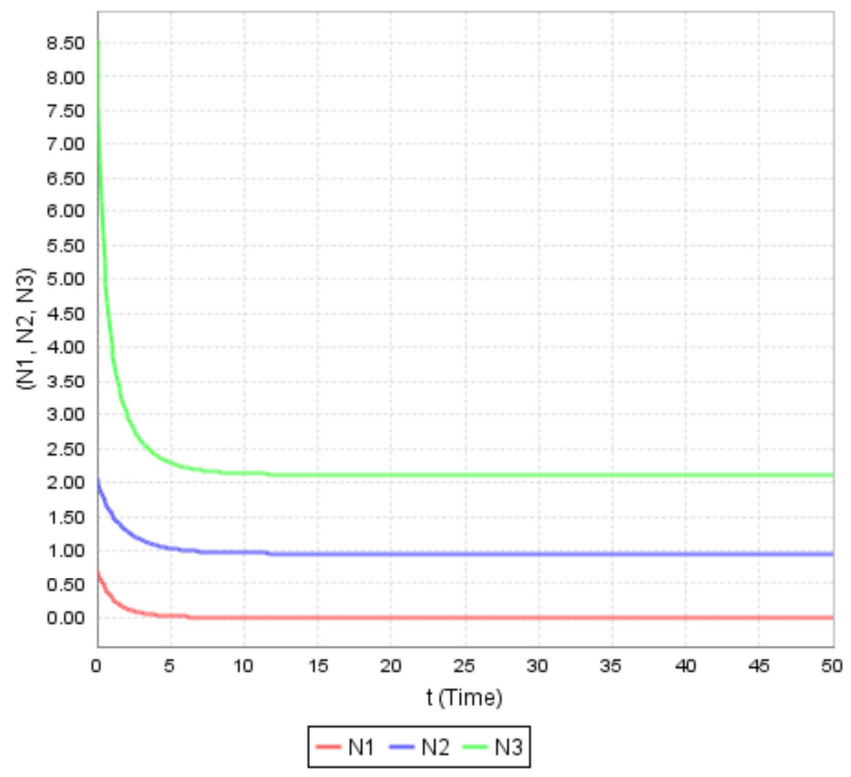

Figure 4. The dynamic behavior of the system with different initial condition and the second and the third species exists while the first species washed out.

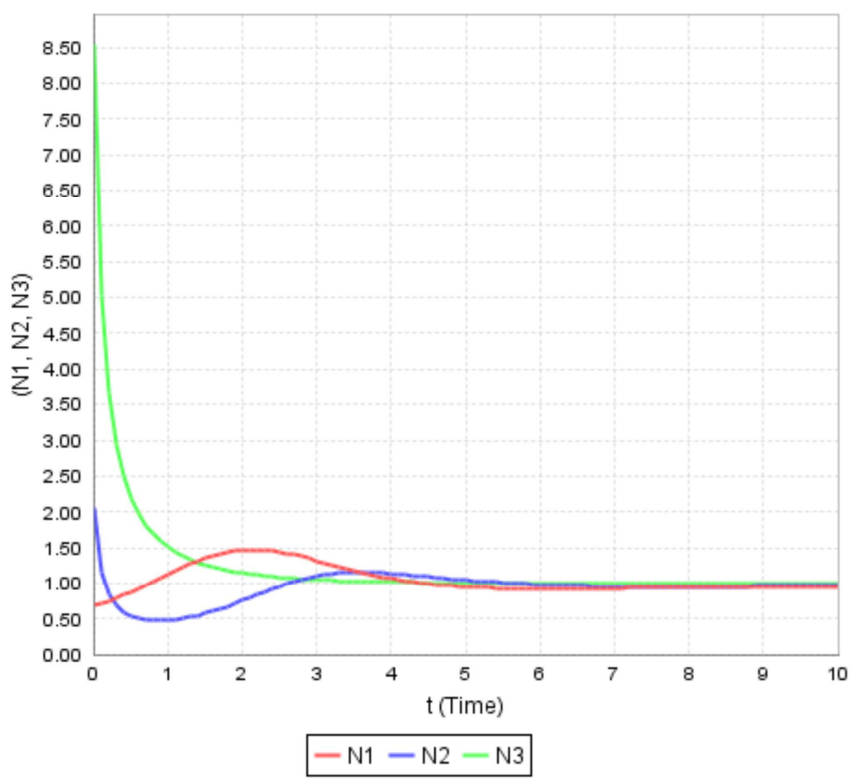

Figure 5. The dynamic behavior of the system with different initial condition and the three species exists. 


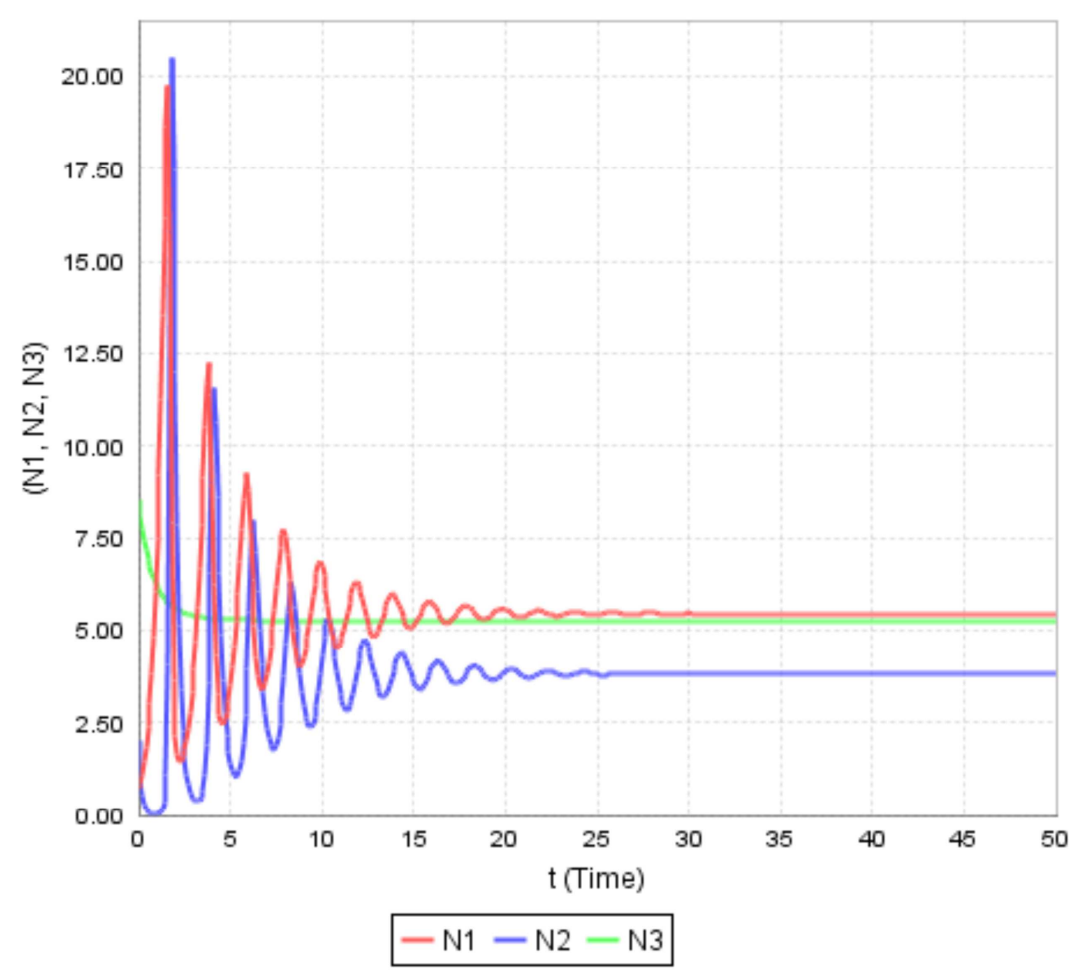

Figure 6. The dynamic behavior of the system with initial condition varying and certain disturbance between the species and living together after certain time $t$.

\section{Conclusion and Future Extension}

In this paper a three species ecosystem with various interactions between the species is considered for investigation. The biological feasible of the system was shown using different mathematical tools, like positivity and boundedness. The equilibrium points are examined. The stability of some the steady states were investigated.

It is observed that among the states, the state in which the first species and the second species washed out (extinct), is asymptotically stable as shown in figure 3 and figure 4 . From this it can be understood that in the former case, though the first and second species are extinct, the third species survives due to non inhibition by its enemy and the response taken between the species. While in the later case, the species continue to exist together because the response taken between the first species and the third species with Monod functional response force them for its survival. The results are illustrated in figures 5 and 6.

In another word we try to incorporate the Monod type response term for the first species of the system given in the model, this seems more interesting and necessary, since more and more species become endangered due to the over exploitation by humans or environmental change. It is shown in figures $3-6$ that the commensalism of the second species to the first species could avoid the extinction of the species. Moreover, if the cooperative intensity is large enough, then the two species could really coexist in a stable state in which the third species could avoid the decline of the other species. However, if the effect of the response of the third species is limited, it has been shown that, the first species still be driven to extinction. In addition to this there is a dispensation or allee effect of the third species, because of over exploitation.

These models can be further extended by other type of response functions and selective harvesting by combined harvesting and also constant rate of harvesting by variable rate of harvesting. This is our further investigation.

\section{Acknowledgements}

The authors would like to thank the editors and anonymous referee for their valuable comments and suggestions which led to a significant improvement of the work.

\section{References}

[1] Lotka A. J., "Elements of physical Biology, William \& Wilking Baltimore, 1925.

[2] Volterra V., Leconssen La Theorie Mathematique De LaLeitte Pou Lavie, Gautheir Villara, Paris, 1931.

[3] Freedman, H. I., "Deterministic Mathematical Models in population Ecology Marcel-Decker, New York, 1980.

[4] Kapur J. N., "Mathematical modeling, Wiley Eastern, 1985.

[5] Meyer W. J., "Concepts of Mathematical modeling”, Mc. Grawhil, 1985.

[6] Paul Colinvaux, Ecology, John Wiley and Sons Inc., New York, 1986. 
[7] Srinivas N. C., "Some Mathematical aspects of modeling in Bio-medical sciences". PhD thesis, Kakatiya university, 1991.

[8] Cushing J. M., Integro-Differnetial Equations and Delay Models in Population Dynamics, Lecture Notes in BioMathematics, 20, Springer Verlag, 1997.

[9] Lakshmi Narayana. K., "A mathematical study of a preypredator ecological model with a partial cover for the prey and alternative food for the predator", PhD thesis, JNTU, 2005.

[10] Lakshmi Narayan. K \& Pattabhi Ramacharyulu N. Ch, 2007, "A prey predator model with cover for prey and alternate food for the predator and time delay", International Journal of Scientific Computing Vol. 1, pg. 7-14.

[11] Ravindra Reddy., "A study on mathematical models of Ecological mutualism between two interacting species" $\mathrm{PhD}$ thesis, O. U, 2008.

[12] Archana Reddy R. Pattabhi Ramacharyulu N. Ch \& Krishna Gandhi. B., "A stability analysis of two competitive interacting species with harvesting of both the species at a constant rate" International Journal of scientific computing (1) pp 57-68, 2007.

[13] Bhaskara Rama Sarma \& Pattabhi Ramacharyulu N. Ch., "Stability analysis of two species competitive ecosystem". International Journal of logic based intelligent systems, Vol. 2 No. 1, 2008.

[14] Seshagiri Rao. N \& Pattabhi Ramacharyulu N. Ch., "Stability of a syn-ecosystem consisting of a Prey -Predator and host commensal to the prey-I" (With mortality rate of prey), 2009.

[15] D. Ravi kiran, B. Rami reddy, N. Ch. P. Acharyulu, "A Numerical analysis of stability of a three species Eco system consisting of Prey, Predator and a third species which is host to prey and enemy to Predator" ANU Journal of Physical Sciences, Volume 3, No. 1 \& 2, 2011.

[16] D. Ravi kiran, B. Rami reddy, 'Stability of a three species ecological system consisting of Prey, predator species and a third species which is a host to the prey and enemy to the predator", Journal of Experimental sciences, Vol. 3, No. 12, 2012.

[17] N. Apreutesei, A. Ducrot, V. Volpert, Competition of species with intra-specific competition, Math. Model. Nat. Phenom., 3, pp. 1-27, 2008.

[18] . H. Malchow, S. Petrovskii, E. Venturino, Spatiotemporal Patterns in Ecology and Epidemiology, Chapman \& Hall/CRC Press, Boca Raton, 2008.

[19] S. G. Ruan, D. M. Xiao, Global analysis in a predator-prey system with nonmonotonic functional response, SIAM J. Appl. Math., 61, pp. 1445-1472, 2001.

[20] R. MacArthur. Geographical Ecology: Patterns in the Distribution of Species. Harper and Row, New York, 1972.
[21] T. W. Schoener. Alternatives to Lotka-Volterra competition: models of intermediate complexity. Theor. Popul. Biol., 1976, 10: 309-333.

[22] N. Apreutesei, G. Dimitriu and R. Strugariu, An optimal control problem for a two-prey and one-predator model with diffusion, Comput. Math. Appl., 67 (2014), $2127\{2143$.

[23] J. L. Bronstein, U. Dieckmann and R. Ferri'ere, Coevolutionary dynamics and the conservation of mutualisms, in Evolutionary Conservation Biology (eds. R. Ferri'ere, U. Dieckmann and D. Couvet), Cambridge University Press, (2004), $305\{326$.

[24] A. E. Douglas, The Symbiotic Habit, Princeton University Press, Princeton, 2010.

[25] Chen, F., Xue, Y., Lin, Q., et al.: Dynamic behaviors of a Lotka Volterra commensal symbiosis model with density dependent birth rate. Adv. Differ. Equ. 2018, 296 (2018).

[26] Cruz Vargas-De-Le'on, Chilpancingo and Guillermo G'omezAlcaraz. Global stability in some ecological models of commensalism between two species. Biomatem'atica 23 (2013), 138-146.

[27] Liu, Y., Xie, X., Lin, Q.: Permanence, partial survival, extinction, and global attractivity of a non autonomous harvesting Lotka Volterra commensalism model incorporating partial closure for the populations. Adv. Differ. Equ. 2018, 211 (2018).

[28] Lin, Q.: Allee effect increasing the final density of the species subject to the Allee effect in a Lotka Volterra commensal symbiosis model. Adv. Differ. Equ. 2018, 196 (2018).

[29] Georgescu, P., Maxin, D.: Global stability results for models of commensalism. Int. J. Biomath. 10 (3), 1750037 (25 pages) (2017).

[30] Liu, Y., Zhao, L., Huang, X., et al.: Stability and bifurcation analysis of two species amensalism model with Michaelis Menten type harvesting and a cover for the first species. Adv. Differ. Equ. 2018, 295 (2018).

[31] Wu, R. X., Lin, L., Zhou, X. Y.: A commensal symbiosis model with Holling type functional response. J. Math. Comput. Sci. 16, 364-371 (2016).

[32] Lei, C.: Dynamic behaviors of a stage structured commensalism system. Adv. Differ. Equ. 2018, 301 (2018).

[33] Yang, L. Y., Xie, X. D., et al.: Dynamic behaviors of a discrete periodic predator prey mutualist system. Discrete Dyn. Nat. Soc. 2015, Article ID 247269 (2015).

[34] Geremew Kenassa Edessa, Boka Kumsa, Purnachandra Rao Koya. Modeling and Simulation Study of the Population Dynamics of Commensal-Host-Parasite System. American Journal of Applied Mathematics. Vol. 6, No. 3, 2018, pp. 97108 . 